\title{
Atraumatic Splenic Rupture after Myocardial Infarction
}

\author{
Muhammad Fahad Arshad, Nasir Javed, Syed Monawer Karim, Ehtasham Ahmad, Noor UI Ain Abid \\ Doncaster Royal Infirmary, Doncaster, United Kingdom
}

Received: 18/12/2017

Accepted: $27 / 12 / 2017$

Published: 01/02/2018

How to cite this article: Arshad MF, Javed N, Karim SM, Ahmad E, Abid NUA. Atraumatic splenic rupture after myocardial infarction. EJCRIM 2018;5: doi:10.12890/2018_000827.

Conflicts of Interests: The Authors declare that there are no competing interests.

This article is licensed under a Commons Attribution Non-Commercial 4.0 License

\section{ABSTRACT}

Atraumatic splenic rupture is a rare but potentially life-threatening event. It mostly happens when the spleen is already diseased; however, sometimes it can be drug induced in a previously normal spleen. Although anticoagulation has been attributed to spontaneous splenic rupture quite frequently, the role of dual antiplatelet therapy is underestimated. We report a case of an 80-year-old woman who developed spontaneous splenic rupture 4 weeks after starting dual antiplatelet therapy.

\section{LEARNING POINTS}

- Atraumatic or spontaneous splenic rupture can be life threatening.

- Various drugs, including granulocyte colony-stimulating factors (GCSF) and anticoagulants, can result in atraumatic splenic rupture in a previously normal spleen.

- Dual antiplatelet therapy can also cause splenic rupture in a previously normal spleen. It can occur as early as a few weeks after initiation of treatment.

\section{KEYWORDS}

Atraumatic splenic rupture, clopidogrel, antiplatelet therapy, myocardial infarction

\section{INTRODUCTION}

Splenic rupture happens mostly after trauma. Although atraumatic or spontaneous cases are not very common, they can be challenging to diagnose, as the absence of a classical history can result in delayed or missed diagnosis ${ }^{[1]}$. Therefore, it is very important for clinicians to consider the potential underlying risk factors that should enable them to select 'at risk' patients and guide further investigations. Among various medications, the role of dual antiplatelet therapy in the aetiology of atraumatic splenic rupture is underestimated. We report the following case, where the initiation of dual antiplatelet therapy resulted in splenic rupture in an elderly patient.

\section{CASE REPORT}

An 80-year-old woman with no previous history of coronary artery disease presented to the emergency department on 28 March 2017 with central chest pain. An ECG done at the time showed anterolateral ST elevation myocardial infarction and troponin levels of $66092 \mathrm{ng} / \mathrm{L}(<2)$. She was therefore referred for immediate primary percutaneous coronary intervention. Unfortunately, she had diffuse coronary artery disease that was not amenable to successful angioplasty. She was thereafter managed with low molecular weight heparin (fondaparinux) for 5 days followed by dual antiplatelet therapy (aspirin and clopidogrel). She was discharged subsequently on 3 April 2017.

She was readmitted on 25 April 2017 with shortness of breath and left-sided pleuritic chest pain along with some left flank pain. Her ECG 
was no different from previous ones and there was no further increase in troponin levels. As an inpatient, she developed severe left-sided abdominal pain. On examination, she was hypotensive and had severe tenderness in her central abdomen. She and her family denied any history of trauma in the preceding weeks.

Her chest X-ray showed small left-sided pleural effusion (Fig. 1) and a CT scan of her abdomen revealed splenic laceration, extensive subcapsular haematoma and free fluid in the abdomen (Fig. 2). She was urgently reviewed by the surgical team. Unfortunately, because of her recent myocardial infarction and moderate to severe left ventricular dysfunction, she was not considered fit for surgery or endovascular therapy, and was therefore managed conservatively. She did not respond to conservative management and remained hypotensive. She was reviewed by the palliative team for pain management and died 4 days later.

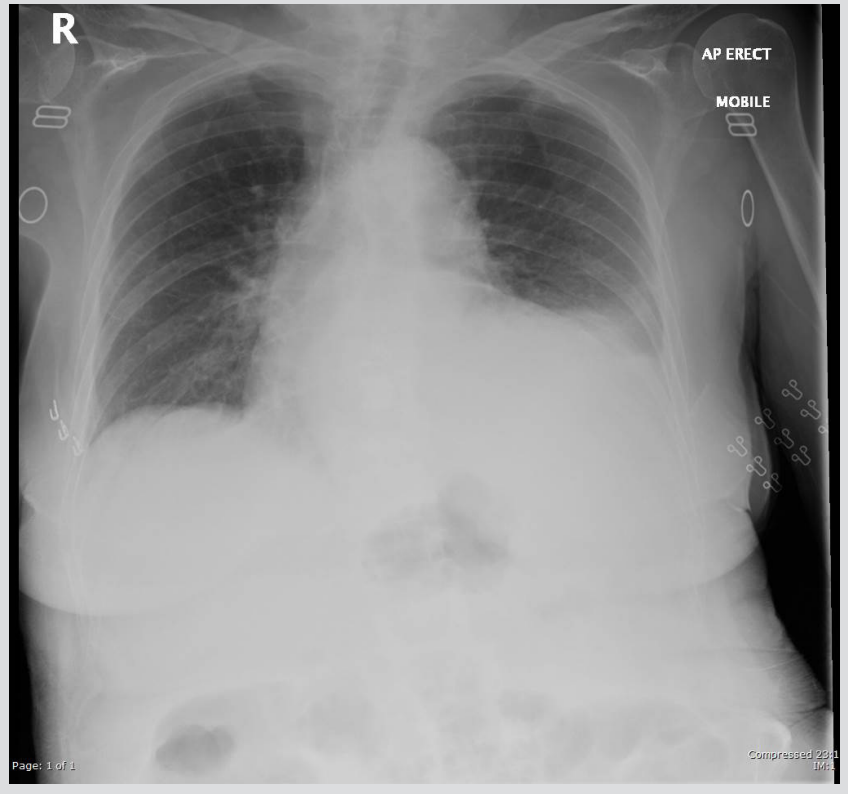

Figure 1. Chest $X$-ray, showing small left-sided pleural effusion

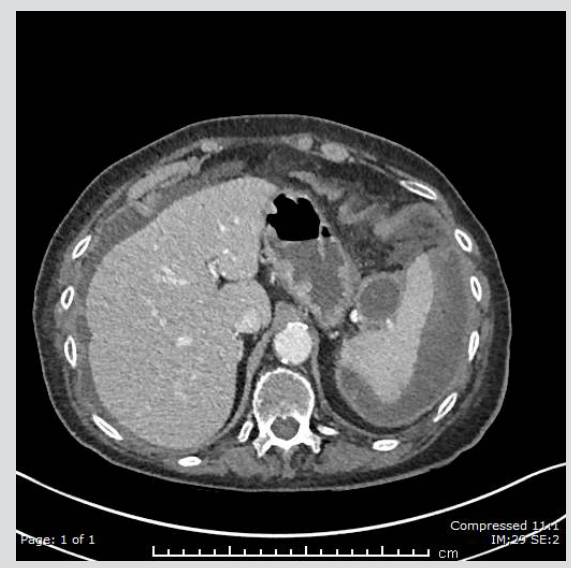

Figure 2. CT scan of abdomen, showing splenic laceration, extensive subcapsular haematoma and free fluid in the abdomen

\section{DISCUSSION}

Splenic rupture is not uncommon after abdominal trauma. In contrast, atraumatic or spontaneous rupture of the spleen is rare, but still can be life threatening. The lack of a previous history of significant trauma can result in delayed diagnosis and poor patient outcomes. A review of the literature for atraumatic splenic rupture identified two systematic reviews consisting of $845^{[1]}$ and $613^{[2]}$ cases each. The first review reported mortality rates of $12.2 \%$ in this cohort of patients. It identified age of more than 40 years, splenomegaly and neoplastic disorders as risk factors associated with high mortality.

Renzulli et al. ${ }^{[1]}$ found that in the vast majority of cases (93\%), despite the absence of compatible history, there were either one or more aetiological factors that predisposed to splenic rupture. In most cases, the underlying cause was not found until after presentation at hospital. All such causes were divided into six major groups, namely (in order of frequency): neoplastic disorders; infectious disorders; inflammatory non-infectious disorders; drug and treatment-related disorders; normal spleen; and mechanical disorders.

Among medications, although there are cases of splenic rupture reported following granulocyte colony-stimulating factors (GCSF), anticoagulants are usually the most common offenders. Several cases of spontaneous splenic rupture have been reported with unfractionated heparin, low molecular weight heparin ${ }^{[3]}$, warfarin ${ }^{[4]}$ and novel oral anticoagulants ${ }^{[5,6]}$ in a previously described normal spleen. As expected, thrombolytic therapy, including both streptokinase and recombinant tissue plasminogen activator ${ }^{[7]}$, have also been implicated. Our literature search identified only two case reports ${ }^{[8,9]}$ attributing dual antiplatelet therapy (aspirin with ticagrelor and aspirin with clopidogrel) to spontaneous splenic rupture. 
The data available in the literature with regard to management of atraumatic splenic rupture is very limited. Treatment options do not differ from cases of blunt abdominal trauma; however, such patients are significantly more likely to undergo splenectomy than if their rupture had been of traumatic aetiology. Renzulli et al.'s review ${ }^{[1]}$ concluded that patients with non-malignant aetiology could be treated conservatively, with organ-preserving surgery or transcatheter arterial embolisation. However, they recommended surgical removal for patients with malignant aetiologies. Overall, the patient's full clinical picture, haemodynamic stability, grade of splenic injury, and the presence or absence of peritonitis should be considered ${ }^{[10]}$. For patients who are not fit for general anaesthesia, conservative or less invasive management is the only option.

Our case highlights the role of antiplatelet therapy and their association with spontaneous splenic rupture in the absence of any other risk factors described above.

\section{REFERENCES}

1. Renzulli P, Hostettler A, Schoepfer AM, Gloor B, Candinas D. Systematic review of atraumatic splenic rupture. Br J Surg 2009;96:1114-21.

2. Aubrey-Bassler FK, Sowers N. 613 cases of splenic rupture without risk factors or previously diagnosed disease: a systematic review. BMC Emerg Med 2012;12:11.

3. Taccone FS, Starc JM, Sculier JP. Splenic spontaneous rupture (SSR) and hemoperitoneum associated with low molecular weight heparin: a case report. Support Care Cancer 2003;11:336-38.

4. de Kubber MM, de Groot B, Kroft LJM. Non-traumatic splenic rupture in a patient on oral anticoagulation. Int J Emerg Med 2013;6:16.

5. Lowry LE, Goldner JA. Spontaneous splenic rupture associated with apixaban: a case report. J Med Case Rep 2016;10:217.

6. Amin A, Safaya A, Ronny F, Islam H, Bhuta K, Rajdeo H. Hemorrhagic shock from spontaneous splenic rupture requiring open splenectomy in a patient taking rivaroxaban. Am Surg 2016;82:e54-5.

7. Revesz E, Grimaldi JA, Clark ET, Podbielski FJ. Spontaneous splenic rupture in a patient receiving thrombolytic therapy. J Vas Bras 2009;8:274-76.

8. Grifoni E, Paniccia R, Giusti B, Sticchi E, Padeletti L, Cavallini C, et al. Non-traumatic splenic rupture on dual antiplatelet therapy with aspirin and ticagrelor after stenting for acute coronary syndrome. J Cardiol Cases 2015;12:65-67.

9. Shaileshkumar GS, Shyamkumar KN, Munawwar A, Vijayan P, Benjamin P. Haemorrhagic shock due to spontaneous splenic haemorrhage complicating antiplatelet therapy: endovascular management. Egypt J Intern Med 2015; 27:154-56.

10. Walker AM, Foley EF. Surgical management of atraumatic splenic rupture. Int Surg J 2016;3:2280-88. 